\title{
Current Perspective on Antithrombin Drugs
}

\author{
John L. McGregor \\ Inserm Unit 331, Faculty of Medicine, RTH Laénnec, University of Lyon 1, Lyon, France
}

\section{Key Words}

Thrombin inhibition - Protease-activated receptor . Thrombin-receptor-activated protease $\cdot$ Crescentic glomerular nephritis · Renal inflammation

\begin{abstract}
Thrombin is a multifunctional serine protease, which is involved in blood coagulation and thrombosis, inflammation and wound repair in tissue injury. Its role in the amelioration of inflammatory tissue injury has been investigated. Protease-activated cell surface receptors (PARs) when activated by thrombin result in the production of proinflammatory mediators. In the kidney, these PARs are expressed on the glomerular epithelium and the vascular endothelium. The significant impact of thrombin inhibition on the development of crescentic glomerular nephritis is discussed.
\end{abstract}

Copyright $(2002$ S. Karger AG, Basel

The following is a brief overview on the potential use of anti-thrombin drugs in new therapeutic areas, distinct from their established use in thromboembolic disorders.

\section{Thrombin}

Thrombin is a serine protease involved in the functioning of a variety of physiological processes, e.g. blood coagulation and thrombosis, inflammation, wound repair in

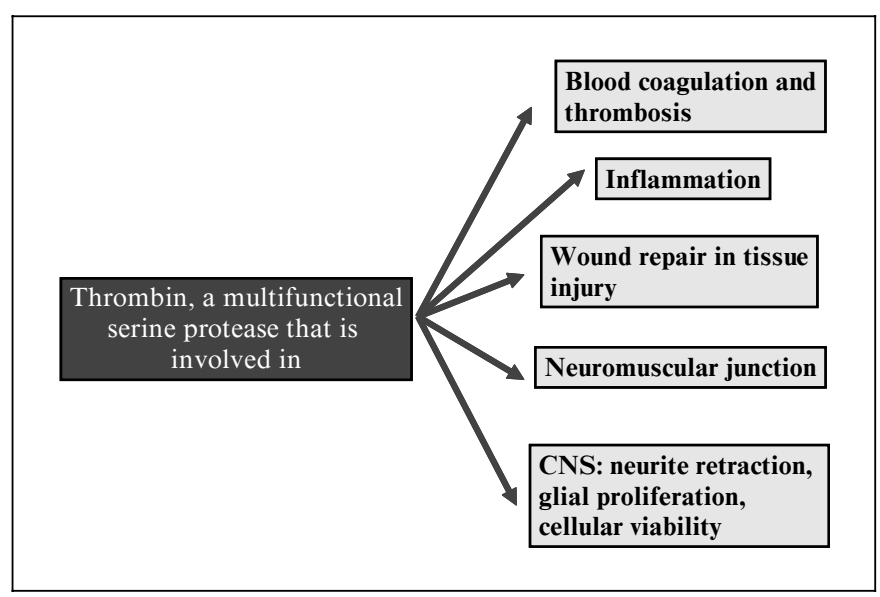

Fig. 1. Processes in which thrombin is involved.

tissue injury, neuromuscular junction activity and, in the central nervous system, neurite retraction, glial proliferation and cellular viability (fig. 1).

Thrombin exerts its effect on both circulating cells and on those in the endothelium of the blood vessels (fig. 2, 3). Platelets are activated to aggregate and synthesise thromboxanes; leucocytes become chemotactic with cytokine production and lymphocytes undergo mitogenesis. In the vessel wall, the influence of thrombin on endothelial cells is to induce activation and expression of cell adhesion molecules (CAM) such as $\mathrm{P}$ and E selectins. In addition, chemokines and autacoids are expressed; and smooth muscle vasoconstriction occurs.

\begin{tabular}{ll}
\hline KARGER & ( 2002 S. Karger AG, Basel \\
1424-8832/02/0329-0029\$18.50/0 \\
$\begin{array}{l}\text { Fax +4161306 12 34 } \\
\begin{array}{l}\text { E-Mail karger@karger.ch } \\
\text { www.karger.com }\end{array}\end{array}$ & $\begin{array}{l}\text { Accessible online at: } \\
\text { www.karger.com/pht }\end{array}$
\end{tabular}

John L. McGregor, PhD, Dr. B.H.

Director of Functional Genomics, Thrombosis Research Institute

Emmanuel Kaye Building, Manresa Road, Chelsea

UK-London SW3 6LR (England)

Tel. +44 207351 8314, Fax +44 207351 8317, E-Mail mcgregor@tri-london.ac.uk 
Fig. 2. Effects of thrombin on circulating and endothelial cells.
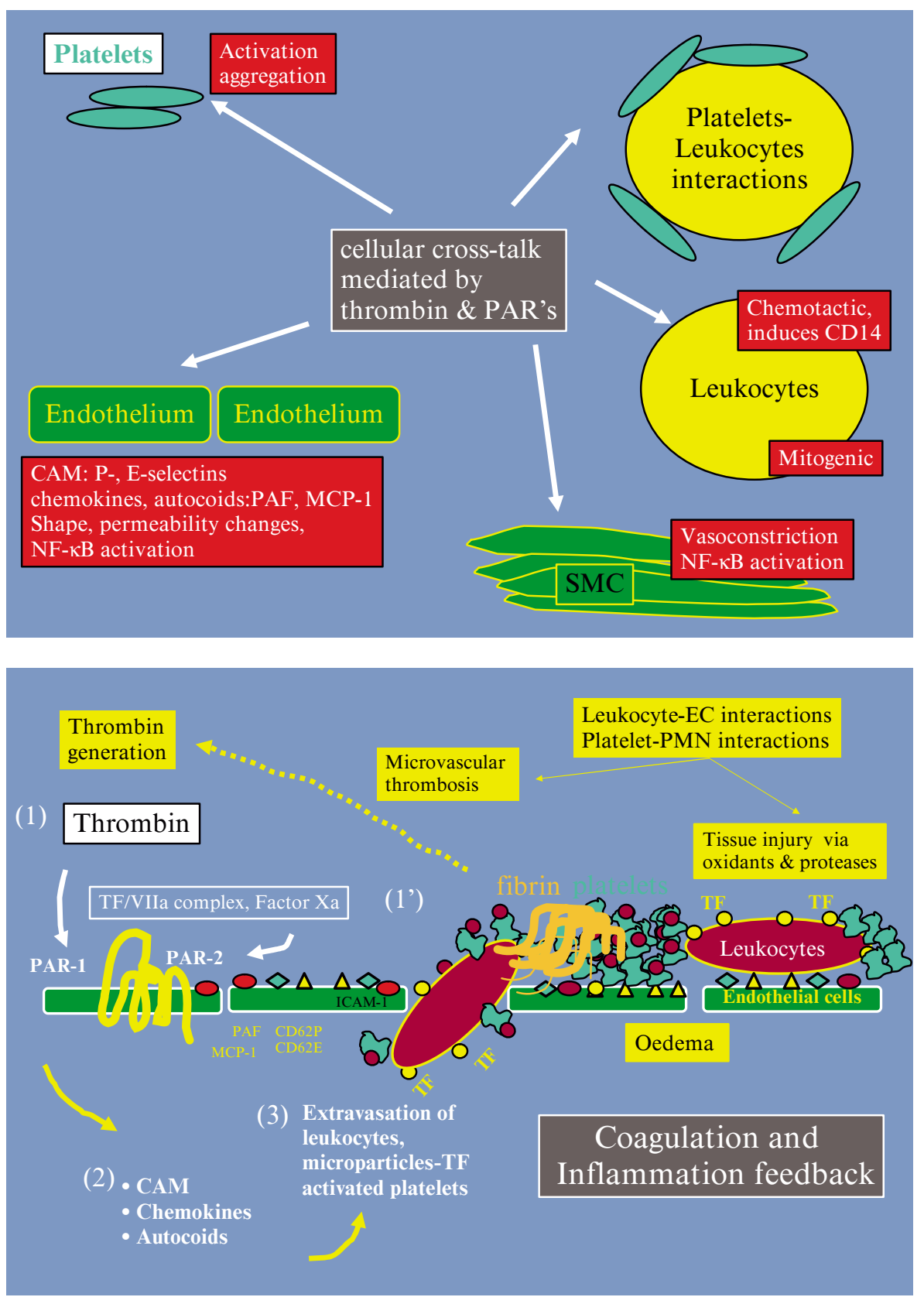

Fig. 3. Thrombin involvement in inflammatory pathways.

These events are brought about via cleavage of thrombin with cell surface protease receptors which become activated (PARs). This mechanism of action affords an understanding of the relationship between the inflammatory and coagulation processes. Thrombin cleaves with PAR-1 and VIIa complex and factor Xa cleave with PAR-2. The presence, together, on the endothelium of activated CAMs with associated selectins leads to leucocyte migration and extravasation. Micro-particles bearing tissue factor plus activated platelets can induce tissue injury. Platelets interacting together will produce microvascular thrombosis and oedema; interaction with leucocytes $(\mathrm{PMN})$ may induce further thrombin generation.

These events make it reasonable to propose that inhibition of thrombin activity on PAR-1 would not only reduce thrombotic sequelae, but also the inflammatory process. The question can thus be asked: can one use as a potential therapeutic target for non-thromboembolic disorders the 'cellular cross-talk' mediated by thrombin and PARs? 


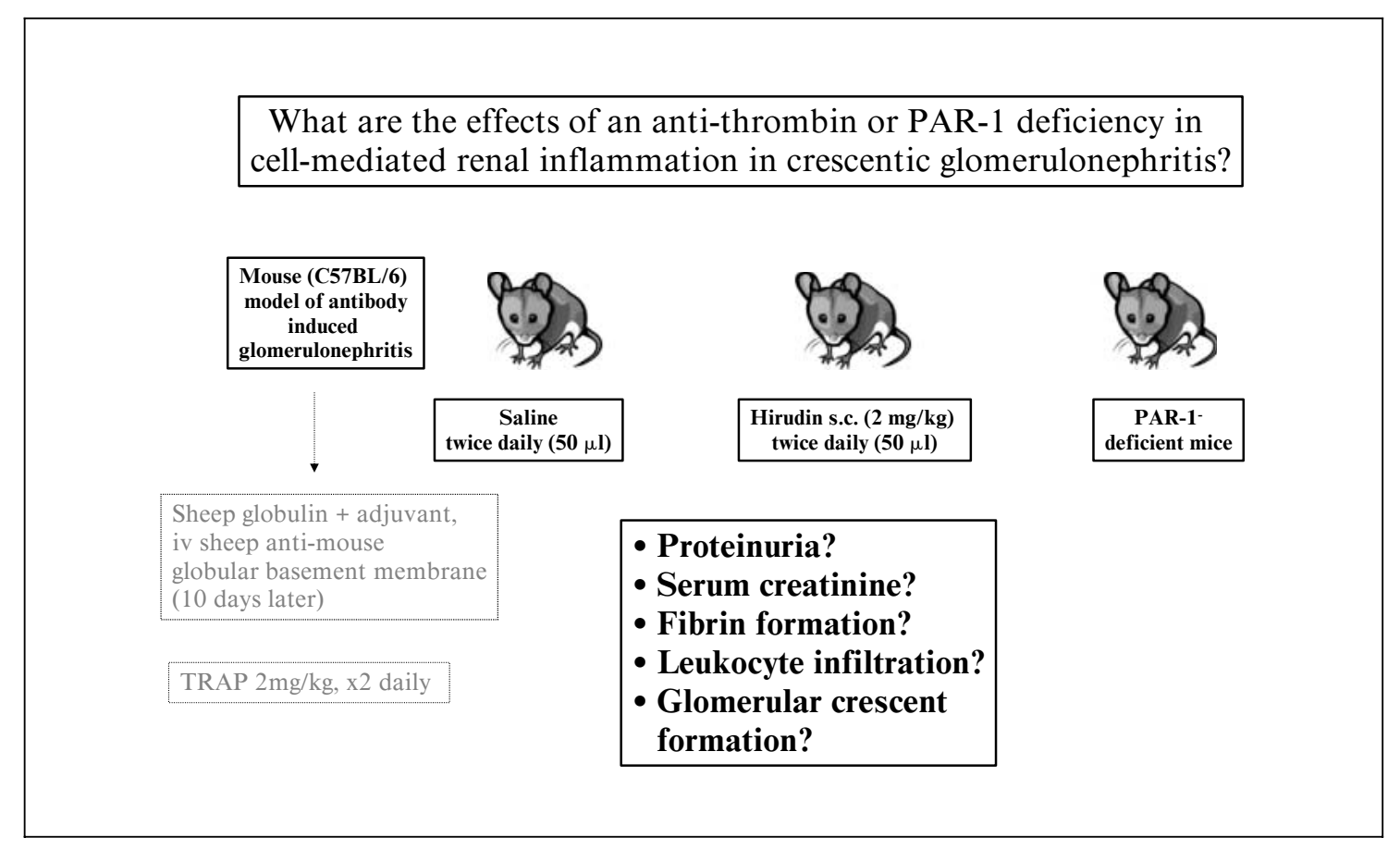

Fig. 4. Thrombin effect on PAR-1 murine model of crescentic GN. From Cunningham et al. [1].

PAR-1 and PAR-2 are expressed in the human kidney: PAR-1 on the glomerular epithelium and endothelium of the renal vasculature. As already stated, activation of PAR-1, by thrombin or thrombin receptor-activating peptides (TRAPs) in vitro, results in the production of proinflammatory mediators from the endothelium. Little is known about the direct pro-inflammatory effects of thrombin in vivo, but it is postulated that the cellular effects of serine proteases of the coagulation pathway may promote inflammatory glomerular injury.

\section{Crescentic Glomerulonephritis}

Glomerulonephritis (GN) is the most frequent cause of end stage renal failure. A particularly severe and rapidly progressive form of this disease is termed crescentic GN. It is characterised by inflammatory cell infiltration at the glomerular level, deposition of fibrin and upregulation of procoagulant molecules. Furthermore, upregulation of PAR-1 messenger RNA is associated with a down-regulation of PAR-1 antigen (fig. 4).

The contribution of thrombin and PAR-1 to inflammatory renal injury has been studied in vivo using a wellcharacterised murine model of crescentic GN [1]. Four groups of WT PAR-1 GKO mice were used. Each group was sensitised by nephritigenic antigen and sheep antimouse glomerular basement membrane globulin to develop glomerulonephritis (fig. 4). One group of mice received normal saline as a control, one group received hirudin to selectively inhibit the serine protease activity of thrombin, one group was PAR-1 deficient and the control group and the PAR-1-deficient mice received, in addition, TRAP, so forming the fourth group.

All mice were treated as above and after 10 days the following parameters were recorded: development of crescentic GN, proteinurea, serum creatinine, fibrin formation, leucocyte infiltration and glomerular crescent formation. Pathology from stained kidney samples was carried out on the four groups of mice to investigate presence of renal tissue damage (fig. 5-7).

It was shown that thrombin is an important mediator of injury in crescentic GN.

Those mice receiving hirudin and those that were PAR-1 deficient exhibited lower levels of proteinurea, serum creatinine, crescent formation, leucocyte and T-cell macrophage infiltration and fibrin deposition. Hirudin, through its blocking action on PAR-1 activity of thrombosis, afforded significant protection from the development of crescentic GN. PAR-1 deficiency protects against de- 

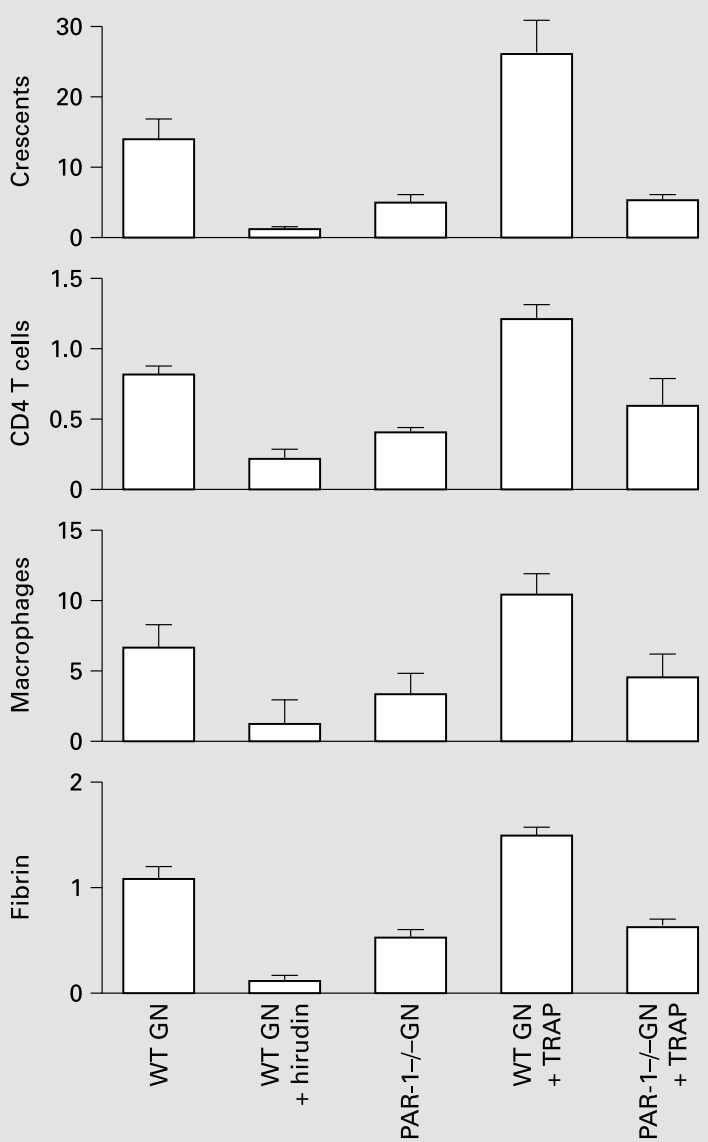
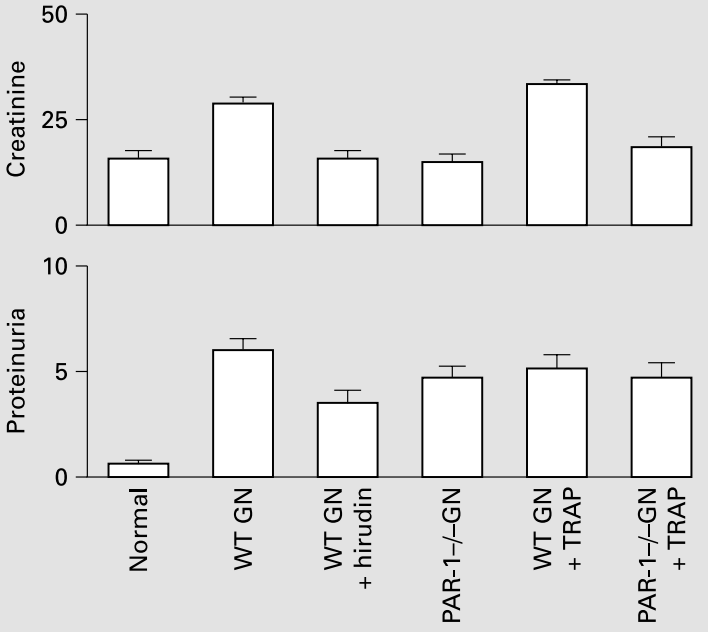

Fig. 5. Effect of thrombin on fibrin, white cells, T cells and crescents in murine model of crescentric GN. From Cunningham et al. [1].

Fig. 6. Effect of thrombin on proteinurea and creatinine in murine model of crescentic GN. From Cunningham et al. [1].

Fig. 7. Involvement of thrombin and PAR-1 in cell-mediated renal inflammation in crescentic GN. From Cunningham et al. [1].

\begin{tabular}{|c|c|c|}
\hline \multicolumn{3}{|c|}{$\begin{array}{l}\text { Thrombin and PAR-1 are implicated in cell-mediated } \\
\text { renal inflammation in crescentic glomerulonephritis }\end{array}$} \\
\hline $\begin{array}{c}\text { Saline } \\
\text { twice daily }(50 \mu \mathrm{l})\end{array}$ & $\begin{array}{c}\text { Hirudin s.c. }(2 \mathrm{mg} / \mathrm{kg}) \\
\text { twice daily }(50 \mu \mathrm{l})\end{array}$ & $\begin{array}{c}\text { PAR-1- } \\
\text { deficient mice }\end{array}$ \\
\hline High & Normal & Normal \\
\hline High & Slightly reduced & Slightly reduced \\
\hline High & Normal & Significantly reduced \\
\hline High & Significantly reduced & Significantly reduced \\
\hline High & Normal & Significantly reduced \\
\hline
\end{tabular}

Serum creatinine

Proteinuria

Fibrin formation

Leukocyte infiltration 

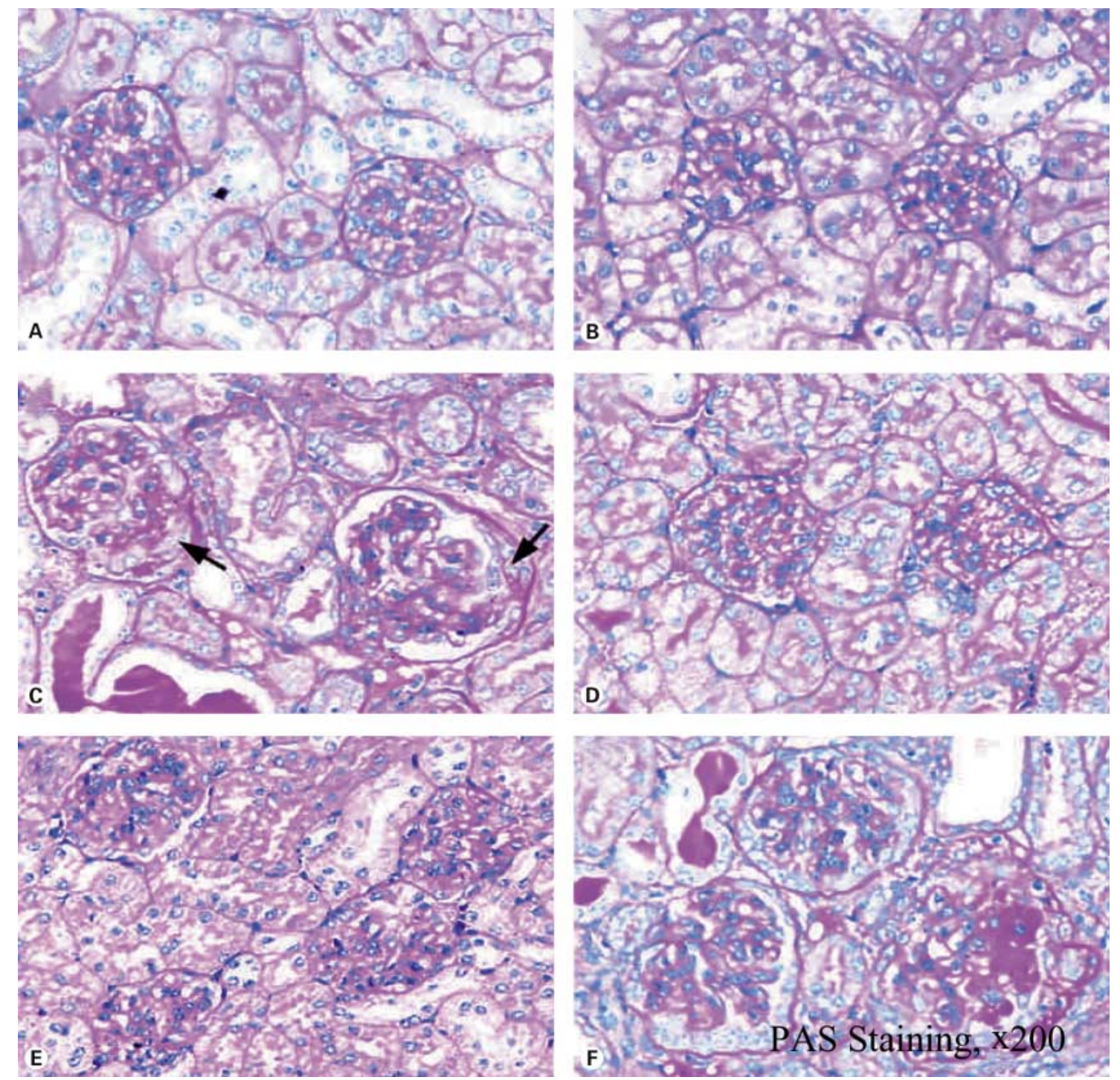

Fig. 8. Effects of thrombin and PAR-1 on glomerular pathology in crescentic GN. From Cunningham et al. [1]. PAS. $\times$ 200. A Glomeruli, control before GN. B Glomeruli in PAR-1-deficient mice before GN. C Following 10 days of GN: proliferative changes in glomerular tufts: crescents, Bowman's space. D Size of glomeruli following 10 days of GN + hirudin. E Following 10 days of GN in PAR-1-deficient mice. F Following 10 days of GN + TRAP.

velopment of crescentic GN (64\% decrease compared with controls).

TRAP given to the control mice increased the levels of these parameters.

Microscopic examination of stained kidney sections showed the following (fig. 8):

A glomeruli before induction of GN are normal;

$\mathrm{B}$ glomeruli from PAR-1-deficient mice before induction of GN are normal;

Current Perspective on Antithrombin

Drugs
C glomeruli from normal controls 10 days after initiation of GN show proliferative changes in the glomerular tufts and crescents in Bowman's capsule;

$\mathrm{D}$ crescentic glomerular injury is markedly diminished by hirudin treatment, although there are still some proliferative changes present;

E PAR-1-deficient mice show marked attenuation of crescentic GN following induction, and

$F$ administration of TRAP accentuates crescentic GN injury. 
9

Fig. 9. Argatroban. From ref. 2.

Fig. 10. Tri-50b. From ref. 2.

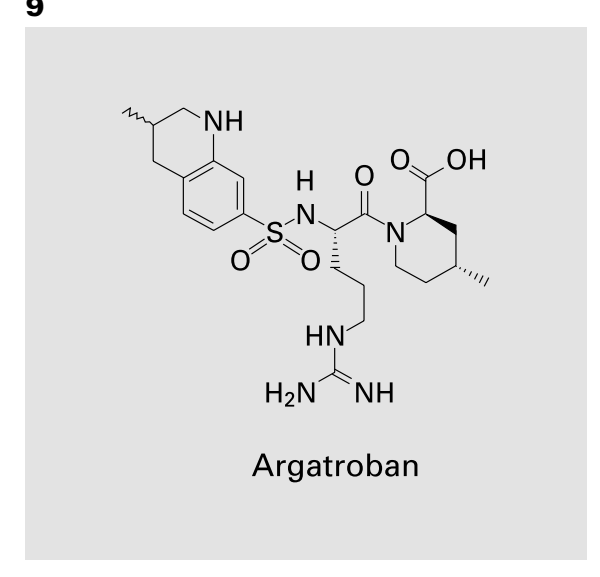

11

Fig. 11. Melagatran. From ref. 2.

Fig. 12. UK-156406. From ref. 2.

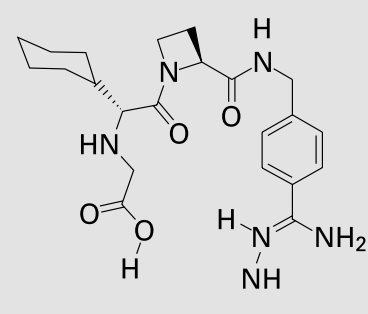

Melagatran
10

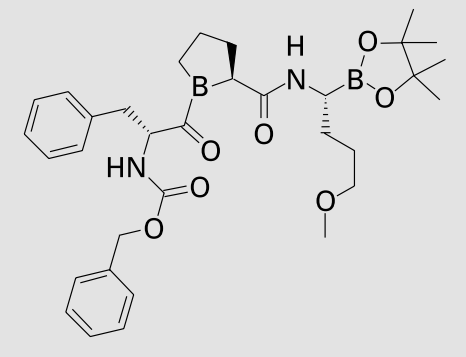

Tri-50b
12

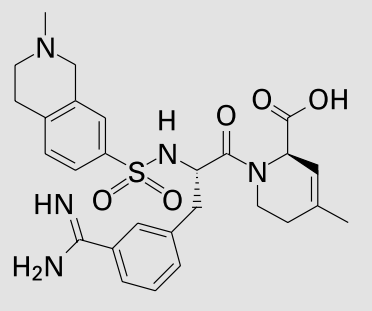

UK-156406
The protective effects of hirudin in this murine model of crescentic GN demonstrate a pivotal pro-inflammatory role for thrombin in the development of inflammatory renal injury. It demonstrates the important contribution of direct cellular effects of procoagulant molecules to inflammatory tissue injury. However, it should be noted that the therapeutic effect of hirudin in reducing renal injury in crescentic GN is not complete. The PAR-1 deficiency data show the importance of this receptor, independent of fibrinogen cleavage or other coagulation factors in the development of renal injury. The TRAP data further confirm the role of PAR-1 in inflammatory-cellmediated disease.

\section{Potential Antithrombin Molecules for Use in Certain Types of Inflammatory Diseases}

The mouse study described indicates that direct inhibition of thrombin PAR-1 cleavage could play an important role in the therapy of some inflammatory diseases.
Argatroban, a Mitsubishi Chemical Industries molecule ( $\mathrm{Ki}$ of $38 \mathrm{n} M$ for human thrombin) (fig. 9), is a reversible direct inhibitor of thrombin, has a short halflife (39-51 min in man), has multiple targets, is non-antigenic and is cleared primarily by the hepatic system.

It is currently available in the USA and Canada (marketed by GlaxoSmithKline) and Korea (marketed by Mitsubishi Pharma Corporation) for the treatment of heparin-induced thrombocytopenia (HIT). In Japan it is marketed by Mitsubishi Pharma Corporation for a number of thrombotic disorders. It could potentially be used in most indications where intravenous heparin is (presently) used.

Some of the new generation of potential thrombin inhibitors, in preclinical development, are claimed to show significant oral bioavailability. A majority of these compounds can be classified as non-electrophilic inhibitors. In clinical development a number of compounds are currently being tested. Tri-50b (fig. 10) is currently in phase II. It is also directed towards arterial and venous thrombosis. It acts as a fast-binding competitive inhibitor 
when compared to S18326 and DuP714. Efegatran (LY2944468) (Eli Lilly) has been tested in patients with unstable angina and in thrombolytic therapy and showed similar efficacy to heparin (a second molecule from Eli Lilly is currently in phase I as an oral anti-thrombin inhibitor). Melagatran (Astra-Zeneca) (fig. 11) is a non-electrophilic inhibitor and is in phase III trials for stroke prevention following atrial fibrillation and in deep vein thrombosis. Beneficial effects of melagatran were observed in renal function and systemic haemodynamics in endotoxaemic pigs with septic shock. Improvement over LMW heparin enoxaparin and heparin in deep vein thrombosis after hip replacement surgery was observed with desirudin (CGP39393, Novartis), a recombinant hirudin.

One of the thrombin inhibitors in clinical development (fig. 12), UK-156406, is an analogue of argatroban and has aroused interest in the treatment of lung fibrosis. Indeed, PARs have been implicated by their role in lung fibrosis. Use of UK-156406 in lung fibrosis suggests that thrombin inhibitors could equally be used as therapeutic tools in areas other than blood coagulation and platelet aggregation.

\section{Conclusion}

Increasing evidence shows that thrombin and PARs are linked to certain types of inflammatory diseases. Thrombin inhibitors may potentially represent a new generation of drugs for the treatment of certain inflammatory disorders, e.g. renal inflammation in crescentic GN.

\footnotetext{
References 1 Cunningham MA, Rondeau E, Chen X, Coughlin SR, Holdsworth SR, Tipping PG: Proteaseactivated receptor 1 mediates thrombin-dependent, cell-mediated renal inflammation in crescentic glomerulonephritis. J Exp Med 2000;191:455-561.

2 Steinmetzer T, Hauptmann J, Sturzebecker J: Advances in the development of thrombin inhibitors. Exp Opin Invest Drugs 2001;10:845864.
}

Current Perspective on Antithrombin Drugs
Pathophysiol Haemost Thromb 2002;32(suppl 3):29-35 